\title{
Synthesis and Characterization of Mesoporous silicon Ellipsoids
}

\author{
Jing Wang ${ }^{1, a}$, Shaodian Shen ${ }^{2, b}$ *, Dongsen Mao, ${ }^{3, c}$ Guanzhong Lu ${ }^{4, d}$ \\ ${ }^{1}$ Research Institute of Applied Catalysis, School of Chemical and Environmental Engineering, \\ Shanghai Institute of Technology Shanghai, P. R.China \\ ${ }^{2}$ Research Institute of Applied Catalysis, School of Chemical and Environmental Engineering, \\ Shanghai Institute of Technology Shanghai, P. R.China \\ ${ }^{3}$ Research Institute of Applied Catalysis, School of Chemical and Environmental Engineering, \\ Shanghai Institute of Technology Shanghai, P. R.China \\ ${ }^{4}$ Research Institute of Applied Catalysis, School of Chemical and Environmental Engineering, \\ Shanghai Institute of Technology Shanghai, P. R.China \\ 1,2,3,4 Haiquan Road 100, Shanghai

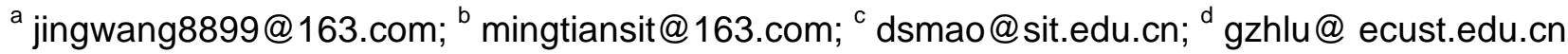

Keywords: anisotropic, magnesiothermic reaction, mesoporous silicon

Abstract. Ellipsoidal silicon nanoparticles were prepared by using the mesoporous silica as raw materials via an magneisothermic reduction reaction with the temperature program control method, while the sample get the corresponding ellipsoidal shape of mesoporous silica. The properties of the ellipsoidal silicon are characterized by scanning electron microscopy, nitrogen adsorption and desoption and X-ray diffraction. The results show that, the sample maintains the original structured mesoporous silica morphology and highly ordered two-dimensional hexagonal structure, while the sample has a high surface area $\left(173 \mathrm{~m}^{2} / \mathrm{g}\right)$, a pore volume $0.39 \mathrm{~cm}^{3} / \mathrm{g}$ and a uniformed pore size of $8.3 \mathrm{~nm}$ after calcination at $650^{\circ} \mathrm{C}$. This properties demonstrate the potential applications in optical sensors and drug delivery.

\section{Introduction}

Mesoporous silicon were widely used in optics, sensors and biomedical for its high surface area, photoluminescence properties and diversity of morphology and structure [1]. Bimbo and co-workers [2] studies shown that mesoporous silica nano-materials as drug delivery agents more easily swallowed by cancer cells, can not through the intestinal wall, have advantages of low toxicity, antioxidant and non-inflammatory response.

As present, low-temperature magnesium thermal reaction has been the most common used method to prepare silicon nanoparticles. Nian Liu and co-workers[3] synthesized the nano-Si by using magnesium as a reducing agent to react with nano silica from the the rice husk, it is demonstrated that the nano-Si with unique small size and porous structure show superior performance as Li-ion battery anode.Richman and co-workers[4] synthesizd mesoporoous silicon with regular structure by using block copolymers KLE as templates to obtain silica membrane, then heated at $675^{\circ} \mathrm{C}$ for $5 \mathrm{~h}$ to carry on the reduction reaction. Bao and co-workers[5] reported that diatomite was reduced to porous silicon at $650^{\circ} \mathrm{C}$ magnesium thermal reaction for $2.5 \mathrm{~h}$. This shows magnesium thermal reaction is usually chosen the melting point of magnesium as reaction temperature, proper control of the reaction conditions to obtain the reduction product of silicon.

In this paper, we report a traditional magnesium thermal reaction to synthesis ellipsoidal silicon, the special mesoporous silica ellipsoid as the raw material, and characterized by SEM, XRD, N2 adsorption-desorption. The obtained material exhibit high surface area and pore volume, special ellipsoidal morphology, and it it promising in fiele of biological, electrical, optical sensors, and drug delivery. 


\section{Experimental section}

\section{Materials}

Poly (propylene oxide)-block-poly (ethylene oxide)-block-poly (propylene oxide) triblock copolymer Pluronic P123 (Mw $=5800$, A.R.) was purchased from Aldrich. Tetraethylorthosilicate (TEOS), hydrochloric acid (36\%-38\%), potassium chloride $(\mathrm{KCl})$, ethanol and magnesium were purchased from Shanghai Chemical Co. All materials were used as received without further purification.

\section{Synthesis}

Mesoporous silica were prepared by hydrothermal method[6]. $0.5 \mathrm{~g} \mathrm{SiO} 2$ was thoroughly ground along with $0.5 \mathrm{~g} \mathrm{Mg}$ powder, and sealed in the special stainless steel reactor, tighten both ends of the nut to completely cut off the air. Placed in a muffle furnace, further heated up to $300^{\circ} \mathrm{C}$ under $\mathrm{N} 2$ atmosphere with a ramping rate of $1^{\circ} \mathrm{C} / \mathrm{min}$ keep for $60 \mathrm{~min}$, then up to $400^{\circ} \mathrm{C}$ keep for $60 \mathrm{~min}$, then up to $500^{\circ} \mathrm{C}$ keep for $60 \mathrm{~min}$, finally up to $650^{\circ} \mathrm{C}$ keep for $120 \mathrm{~min}$. After cooling down, the obtained powders were soaked in $1 \mathrm{M} \mathrm{HCl}$ solution for $6 \mathrm{~h}$, to remove $\mathrm{MgO}$ and unreacted $\mathrm{Mg}$ powder, and then they were soaked in 5\% hydrofluoric acid for $10 \mathrm{~min}$ to ensure that any unreacted or newly formed $\mathrm{SiO} 2$ was removed. Then washing with deionized water and drying under vacuum at room temperature to obtain the mesoporous Si powders.

\section{Characterization}

Scanning electron microscopy (SEM) imageswere taken by a Hitachi S3400 scanning electron microscope.Small angle X-ray scattering (XRD) measurements were taken on a PANalytical X'Pert diffractometer using $\mathrm{Cu} \mathrm{Ka}$ radiation $(40 \mathrm{kV}, 40 \mathrm{~mA})$. Nitrogen adsorption / desorption isotherms were measured at $77 \mathrm{~K}$ using a Micromeritics ASAP 2020 system. Specific surface areas of the samples were determined by nitrogen adsorption data in the relative pressure range from 0.05 to 0.2 using the Brunauer-Emmett-Teller (BET) method. Pore size distributions and the pore volumes were calculated by the adsorption branches of isotherms by the Barrett-Joyner-Halenda (BJH) method, and the total pore volumes were determined from the adsorbed amount at a relative pressure $\mathrm{P} / \mathrm{P} 0$ of 0.99 .

\section{Results and discussion}

The SEM image of mesoporous silica (Fig. 1a) and the reduced product of mesoporous silicon (Fig. 1b) shows that after magnesium thermal reduction reaction, the morphology of mesoporous silicon remains, and there is no sintering phenomenon. It is demonstrated that the method of controlling the heating rate can effectively maintain the morphology of the sample. At the same time, by measuring the mesoporous ellipsoid size before and after calcining can be seen, in front of the thermal reduction of magnesium, the major axis of mesoporous silica ellipsoid is $1.44 \mathrm{um}$, a minor axis is $0.81 \mathrm{um}$, has an aspect ratio of 1.94. After magnesium thermal reduction reaction, the mesoporous silica ellipsoid has a major axis of $1.09 \mathrm{um}$, a minor axis of $0.55 \mathrm{um}$, aspect ratio of 1.98. It shows that in high-temperature roasting process, mesoporous silica material has the overall contraction of morphology caused by pores contraction, which was $24 \%$ shrinkage of the longitudinal direction and $32 \%$ shrinkage rate of the short axis direction. This difference is happened because of that the pore channel of mesoporous silica is ordered along the longitudinal direction, thus the firing process by gravity collapse in the short axis of silica get some more.
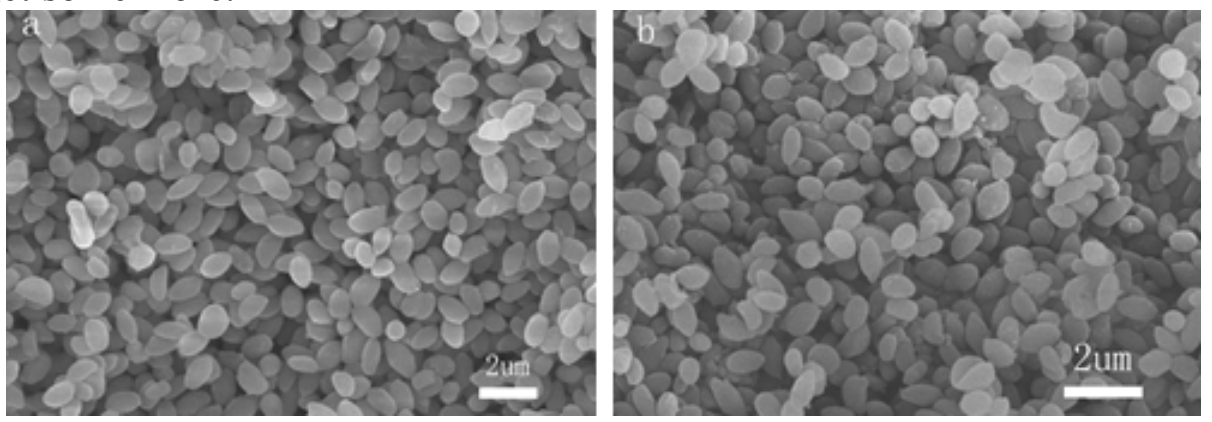

Fig.1 SEM images of (a) ellipsoidal mesoporous silica and (b) mesoporous silicon ellipsoids 
Wide angle XRD patterns of $\mathrm{MgO} / \mathrm{Si}$ and mesoporous $\mathrm{Si}$ are shown in Fig. 2. Seen from the Figure 2a, there are typical diffraction peak of cubic phase of $\mathrm{MgO}\left(2 \theta=36.91^{\circ}, 42.91^{\circ}, 62.27^{\circ}, 74.70^{\circ}\right.$, $78.59^{\circ}$, JCPDS 45-0946) and mesoporous $\mathrm{Si}\left(2 \theta=28.54^{\circ}, 47.39^{\circ}, 56.19^{\circ}, 69.21^{\circ}, 76.48^{\circ}\right.$, JCPDS27-1402) of sample $\mathrm{MgO} / \mathrm{Si}$. After the removal of $\mathrm{MgO}$, only the peaks of mesoporous $\mathrm{Si}$ indexed as (111), (220), (311), (400) and (331) reflaction. And the intensities of diffraction peaks slightly increased, peak width slightly sharp. It is consistent with the expected results.

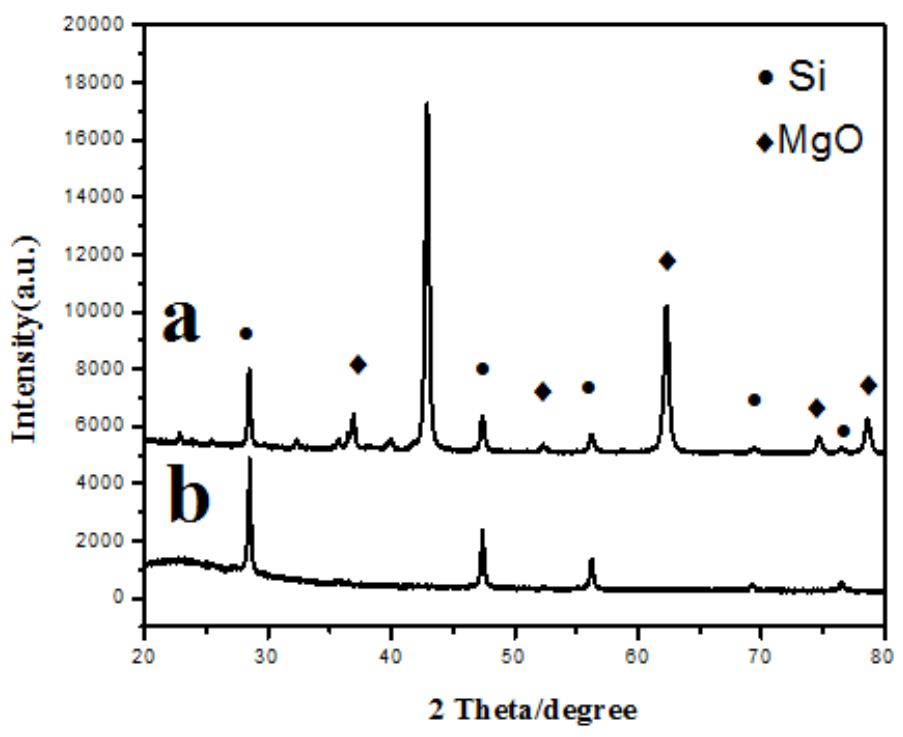

Fig. 2 Wide-angle XRD patterns of (a) $\mathrm{MgO} / \mathrm{Si}$ composites and (b) mesoporous silicon

Fig. 3 gives the small angel XRD patterns of mesoporous $\mathrm{SiO} 2, \mathrm{MgO} / \mathrm{Si}$ and mesoporous $\mathrm{Si}$. It can be seen that the mesoporous structure still remain after the $650^{\circ} \mathrm{C}$ magnesium thermal reaction. Due to the presence of $\mathrm{MgO}$ in the mixture of $\mathrm{MgO} / \mathrm{Si}$, the diffraction peaks become relatively weaker.

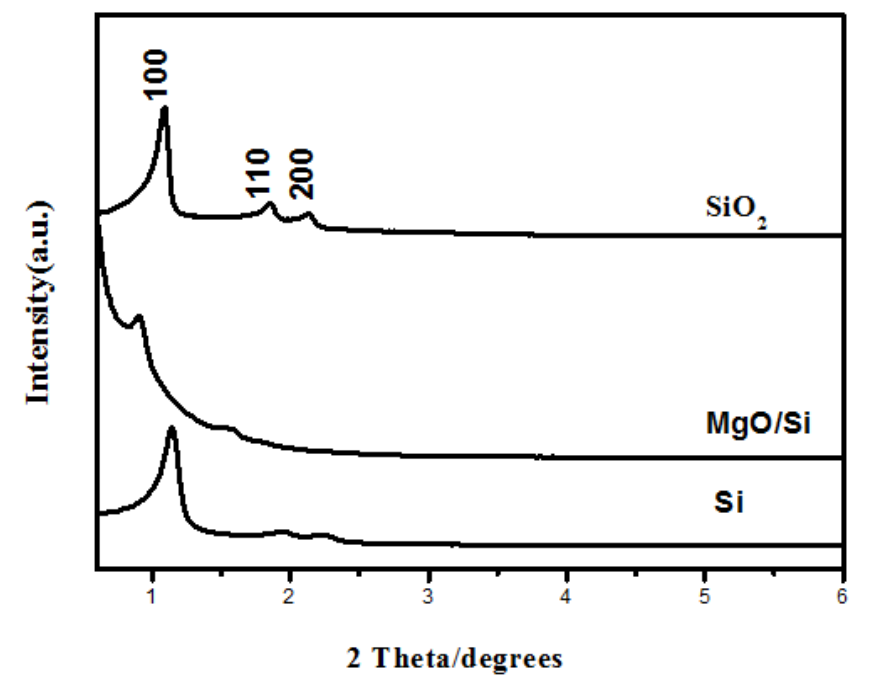

Fig. 3 Small-angle XRD patterns of mesoporous silica with ellipsoidal morphology,

$\mathrm{MgO} / \mathrm{Si}$ composites and mesoporous silicon ellipsoids

Fig. 4 shows the $\mathrm{N}_{2}$ sorption isotherms and BJH pore size distribution plots of mesoporous $\mathrm{Si}$, the isotherms exhibit the same type IV adsorption behavior, meanwhile, a H1-type hysteresis loop is present at the relative pressure of 0.4-1.0 with an obvious capillary condensation, indicate of mesoporous structure. The surface area of $\mathrm{Si}$ is $173 \mathrm{~m}^{2} \mathrm{~g}^{-1}$, pore volume is $0.39 \mathrm{~cm}^{3} \mathrm{~g}^{-1}$. 


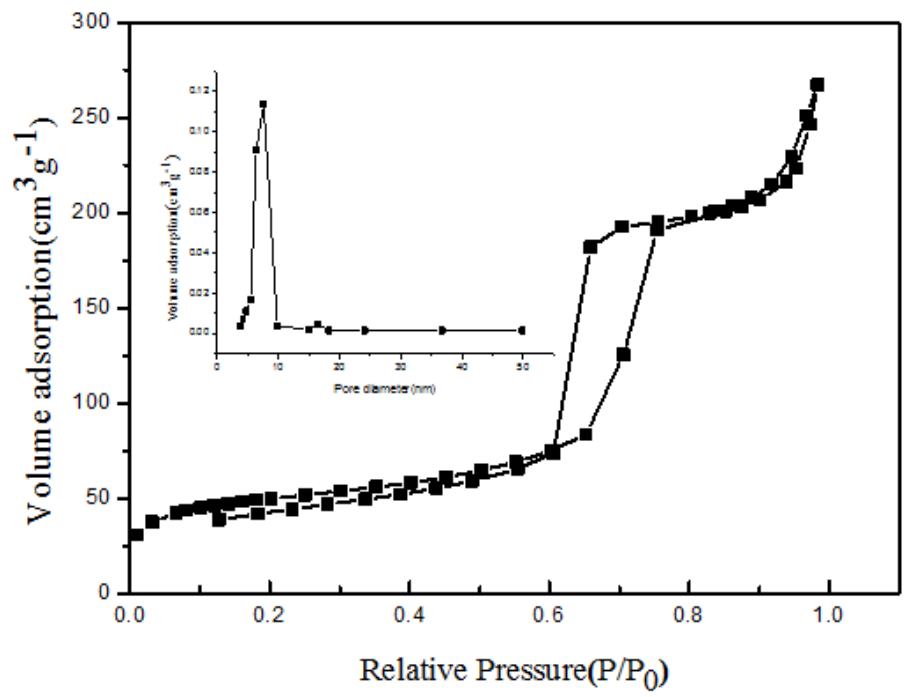

Fig. 4 Nitrogen adsorption-desorption isotherms and BJH pore size distribution of mesoporous silicon ellipsoids

\section{Conclusions}

Using a simple magnesium thermal reaction theory magnesium, mix special ellipsoid shape mesoporous silica with $\mathrm{Mg}$ power, control the program heating rate, at the temperature of $650^{\circ} \mathrm{C}$ to obtain the mesoporous Si with a special ellipsoidal morphology. This synthetic method is simple, can be used in the field of drug delivery and photoluminescence et al.

\section{Acknowledgments}

We are grateful for financial support from the National Science Foundation of China (20971087/B0101), Technology Commission of Shanghai Municipality (08ZR1418800) and Education Commission of Shanghai Municipality (12YZ167)

\section{References}

1. Obrovac M N, Krause L J. Journal of The Electrochemical Society, 2007, 154 (2): A103-A108.

2. Bimbo, L. M., ACS nano, 2010, 4 (6): p. 3023-3032.

3. F E, CA T, CW C, et al. Bioconjugate Chemistry, 2011, 22 (6):1081-1088.

4. Miska P, Dossot M, Nguyen T D, et al. Journal of Physical Chemical C, 2010, 114 (41): 17344-17349.

5. Bimbo L M. Acs Nano, 2010, 4(6):3023-3032. 